\title{
NP-TECHNIQUE AS A TOOL IN DECISION MAKING
}

\section{ERKKi Pesonen}

1. It is likely that in the future applications of actuarial methods to the decision making in non-life companies will more and more relate to the utility concept as was proposed by $\mathrm{K}$. Borch $[\mathrm{I}]$ about fifteen years ago. In this connection it will be important to have workable numerical methods. The calculation of the distribution function of the profit is an unavoidable problem from a practical point of view. Even if it is possible to compute this function today accurately with computers by using the ingenious technique developed by $\mathrm{H}$. Bohman [2], integrals become very laborious when applied to the decision making procedure based on utility concepts. This paper intends to show that the NP-technique,--proposed for the first time by L. Kauppi and P. Ojantakanen in actuarial science [3]--, is particularly suitable in integrals needed for utility calculations.

2. Let $F(x)$ be the distribution function of the total amount of claims and let its mean, standard deviation, skewness and kurtosis be respectively $m, \sigma, \gamma_{1}$ and $\gamma_{2}$. The NP-technique uses the system of equations $\int F(x)=\Phi(y)$

$\left\{\frac{x-m}{\sigma}=y+\frac{1}{6} \gamma_{1}\left(y^{2}-\mathrm{I}\right)+\left[\frac{1}{24} \gamma_{2}\left(y^{3}-3 y\right)-\frac{1}{36} \gamma_{1}^{2}\left(2 y^{3}-5 y\right)\right]\right.$

where $\Phi(y)$ is the standardized normal distribution function. If the parameters $m, \sigma, \gamma_{1}$ and $\gamma_{2}$ and $F(x)$ are known, $y$ is directly found from the tables of the normal distribution function, and thereafter the second equation directly gives the value of $x$. If, vice versa, $x$ and the above parameters are known, $F(x)$ is obtained by solving $y$ from the second equation ( $\mathrm{I}$ ), or, more practically, by using the converted NP-expansion instead of (I), i.e. [4]:

$$
\begin{aligned}
& \left\{\begin{array}{l}
F(x)=\Phi(y) \\
y=z-\frac{1}{6} \gamma_{1}\left(z^{2}-\mathrm{I}\right)+\left[\frac{1}{36} \gamma_{1}^{2}\left(4 z^{3}-7 z\right)-\frac{1}{24} \gamma_{2}\left(z^{3}-3 z\right)\right.
\end{array}\right. \\
& \text { where } z=\frac{x-m}{\sigma}
\end{aligned}
$$


Sometimes it is sufficient to use short forms of the formulae (I) and (2), obtained by omitting the terms in the brackets. If these rougher approximations are used, the estimation of the kurtosis $\gamma_{2}$ remains unnecessary.

3. The NP-formulae are often very practical in connection with integrals involving the distribution function $F(x)$ as well as in the direct numerical calculation of these equations. An example is the explicit formula for the Stop Loss premium given in [5]. Another example, given in [6] as well as other developments presented in this paper, relates to the upper limit of the probability of ruin. It is well-known that under certain conditions this upper limit is $e^{-R U}$, where $U$ is the initial capital of the company and the constant $R$ is obtained from the equation

$$
e^{(1+\lambda) m R}=\int_{0}^{\infty} e^{R x} d F(x) \quad \text { ( } \lambda \text { safety loading). }
$$

If the short form of $(\mathrm{I})$ is used in the integral $\int_{0}^{\infty} e^{R x} d F(x)=$ $\int_{-\infty}^{+\infty} e^{R x} d \Phi(y)=\frac{I}{\sqrt{2 \pi}} \int_{-\infty}^{+\infty} e^{R x-1 / 2 y^{2}} d y$, the result becomes

$m R+\frac{1}{2} \log (\mathrm{I}-s R)=-\frac{1}{2} s R+\frac{R^{2} \sigma^{2}}{2(\mathrm{I}-s R)} \quad\left(s=\frac{\sigma}{3} \gamma_{1}\right)$

This equation is very quickly solved by iteration, starting with the value $R$ obtained by use of the approximation $\log (I-s R) \approx$ $-s R$.

4. As an example of how the NP-technique can be used in utility calculations let us consider the distribution function of the capital, say $X$, at the end of the fiscal year. If the total amount of the claims during this year is $x$, the rate of interest $i$, then approximately

$$
X=U(\mathrm{I}+i)+(\mathrm{I}+\lambda) m \sqrt{\mathrm{I}+i}-x \sqrt{\mathrm{I}+i}
$$

and hence the distribution function of the capital $X$, say $G(X)$, becomes

$$
G(X)=\mathrm{I}-F\left(U \sqrt{\mathrm{I}+i}+(\mathrm{I}+\lambda) m-\frac{\mathrm{I}}{\sqrt{\mathrm{I}+i}} X\right) .
$$


Now suppose that the range of $X$ is divided into a finite number of intervals and suppose that the utility function $u(X)$ the company attaches to the capital $X$ is in each of the intervals a polynomial.

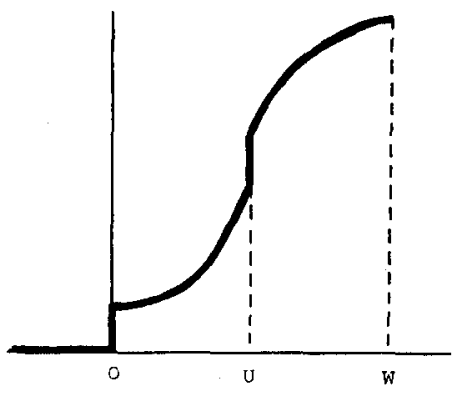

Fig. I

An example is given in Figure I where the utility function is supposed to be constant below the ruin point $X=0$, a polynomial between the ruin point $X=0$ and the loss point $X=U$, and another polynomial between the loss point $X=U$ and the maximum profit point, say $X=W$. In the ruin point and in the loss point the utility function may or may not have steps.

5. Let now one of the above intervals where the utility function can be presented as a polynomial be $(\alpha, \beta)$. Hence it is supposed that in the interval $(\alpha, \beta) u(X)=C_{0}+C_{1} X+\ldots+C_{N} X^{N}$. The use of the formulae

$\left\{\begin{array}{l}\int_{A}^{B} y^{2 k+1} d \Phi(y)=\Phi^{\prime}(A) \sum_{n=0}^{k} A^{2 n}-\Phi^{\prime}(B) \sum_{n=0}^{k} B^{2 n} \\ \int_{A}^{B} y^{2 k} d \Phi(y)=\Phi(B)-\Phi(A)+\Phi^{\prime}(A) \sum_{n=1}^{k} A^{2 n-1}-\Phi^{\prime}(B) \sum_{n=1}^{k} B^{2 n-1}\end{array}\right.$

will be made in the following developments. In order to simplify notations suppose that the value of $x$ obtained from the second Equation (I) is inserted in the right hand side of Equation (4) and write the result simply

$$
X=a_{0}+a_{1} y+a_{2} y^{2}+a_{3} y^{3} .
$$


According to the preceding assumption in the interval $(\alpha, \beta)$ the utility function can be presented as a function of $y$, $u(X)=C_{0}+C_{1} X+\ldots+C_{N} X^{N}=D_{0}+D_{1} y+\ldots+D_{3 N} y^{3 N}$, say.

Of course, if the short form of the $N P$-expansion is used, $a_{3}=0$, and instead of $3 N$ in the above equation the value $2 N$ should be written.

Since evidently $d G(X)=-d F(x)$, it is possible to make use of equations (5) in the following way:

$$
\begin{aligned}
\int_{\alpha}^{\beta} u(X) d G(X)= & \\
-\sum_{n=0}^{3 N} \int_{y(\alpha)}^{v(\beta)} D_{n} y^{n} d \Phi(y) & =[\Phi(A)-\Phi(B)]\left(D_{0}+D_{2}+\ldots\right) \\
& +\left[\Phi^{\prime}(B)-\Phi^{\prime}(A)\right]\left(D_{1}+D_{3}+\ldots\right) \\
& +\left[\Phi^{\prime}(B) B-\Phi^{\prime}(A) A\right]\left(D_{2}+D_{4}+\ldots\right) \\
& +\left[\Phi^{\prime}(B) B^{2}-\Phi^{\prime}(A) A^{2}\right]\left(D_{3}+D_{5}+\ldots\right) \\
& +\ldots,
\end{aligned}
$$

where $A$ (respectively $B$ ) is obtained from $A=y(\alpha)$ (respectively from $B=y(\beta))$ by inserting first $\alpha$ (resp. $\beta$ ) in place of $X$ in equation (4). Then $x$ is solved from this equation and the result is inserted in the second equation (2) giving directly $y(\alpha)$ (resp. $y(\beta)$ ).

6. Accordingly the integral $\int_{\alpha}^{\beta} u(X) d G(X)$ can be explicitly calćulated in each of the given intervals $(\alpha, \beta)$. The final result, the utility of the decision leading to the distribution $G(X)$, i.e.

$$
U(G)=\int_{-\infty}^{+\infty} u(X) d G(X)
$$

is then obtained by simply summing up all the integrals

$$
\int_{\alpha}^{\beta} u(X) d G(X) \text {. }
$$

The above procedure is fairly simple and enables us to calculate the result without computers if there is only a limited number of alternative decisions to hand. 
7. Of course in practical decision situations the management of an insurance company does not usually base its decisions only on a simple one-dimensional utility function $u(X)$. A more gencral approach, based on a multidimensional utility function $u(X$; $\left.p_{1}, p_{2}, \ldots, p_{r}\right)$, is equally easily computed following the above technique as soon as the parameters $p_{i}$ (e.g. annual premiums, loading for administration costs or any matters the management considers important) can be considered to be sufficiently nonstochastic, and preferably to be possible to include as parameters of the above constants $C_{i}$ only.

\section{References}

1] Borch, K., The Utility Concept Applied to the Theory of Insurance. ASTIN Bull., I96I.

[2] BohmaN, H., From Characteristic Function to Distribution Function via Fourier Analysis, BIT 12, 1972.

[3] Kauppi, L. and Ojantakanden, P'., Approximations of the Generalized Poisson Function, ASTIN Bull., 1969.

[4] Pörn, K., A Study in Risk Theory and its Application to the Computation of the Fluctuation Reserve used in Finland, Skand. Aktuarietidskrift, I 968.

[5] Pesonen, E., NP-approximation of Risk Processes, Skand. Aktuarietidskrift, I968 and Erratum, Skand. Aktuarietidskrift, 1969.

[6] Pesonen, E., Kikenriron to sono oyō, Tokyo 1973. 